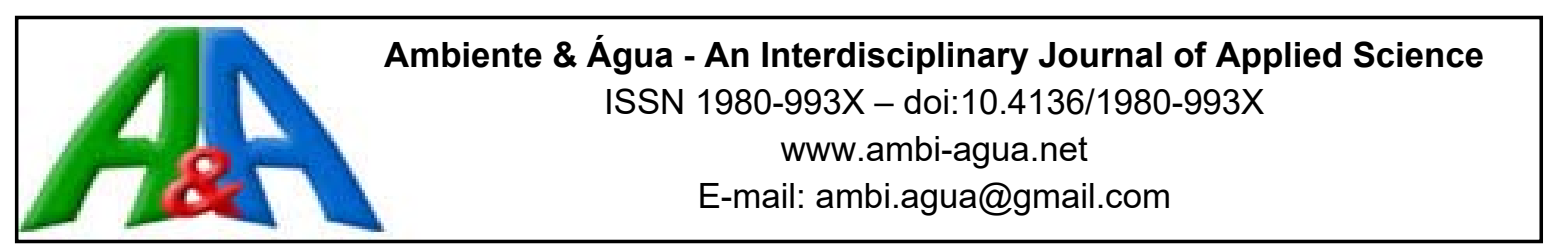

\title{
Evaluación del riesgo a la contaminación de los acuíferos de la Reserva Biológica de Limoncocha, Amazonía Ecuatoriana
}

\author{
doi:10.4136/ambi-agua.2030
}

Received: 20 Oct. 2016; Accepted: 15 May 2017

\section{Andrea Elizabeth Jarrín'; José Gabriel Salazar; Miguel Martínez-Fresneda Mestre ${ }^{1,2^{*}}$}

${ }^{1}$ Universidad Internacional SEK, Quito Pichincha, Ecuador Facultad de Ciencias Naturales y Ambientales ${ }^{2}$ Universidad Internacional SEK, Providencia Región Metropolitana, Chile Dirección de Investigación

*Corresponding author: e-mail: mestre.martinez@uisek.edu.ec, andy_j24@hotmail.com, jose.salazar@uisek.edu.ec

\section{RESUMEN}

El agua subterránea es una de las principales fuentes de abastecimiento para los habitantes de las parroquias de Limoncocha y de Pompeya, ubicadas en la Amazonía ecuatoriana donde, además, se encuentra la Reserva Biológica Limoncocha (RBL) y su área de influencia (AI), cuyo ecosistema subterráneo recibe un aporte de agua proveniente de flujos externos a su límite. Tradicionalmente, el agua subterránea ha sido considerada protegida por el subsuelo, sin embargo, la disposición de contaminantes estables en zonas de infiltración favorable, podría causar que este recurso se contamine, poniendo en riesgo la salud de la población y la sostenibilidad de la reserva. Por ello, se propuso evaluar el peligro de contaminación del agua subterránea de dicha reserva, a través de la superposición de un mapa de vulnerabilidad del acuífero aplicando el método GODS, con un inventario de cargas contaminantes usando el método POSH y en función a esto, priorizar medidas en la protección de este recurso. Se determinó que, en la mayor parte del área de estudio, no existe peligro de contaminación del agua subterránea, no obstante, también se encontraron zonas de moderado y alto peligro para las que se propusieron medidas para prevenir el deterioro de la calidad del agua subterránea.

Palabras clave: acuíferos, agua subterránea, contaminación del agua subterránea, sistemas de información geográfica.

\section{Avaliação do risco à contaminação dos aquíferos da Reserva Biológica de Limoncocha, Amazônia Equatoriana}

\section{RESUMO}

A água subterrânea é uma das principais fontes para o abastecimento dos habitantes das paróquias de Limoncocha e de Pompeya, localizadas na Amazônia equatoriana, onde se encontra a Reserva Biológica Limoncocha (RBL) e sua área de influência (AI), cujo ecossistema subterrâneo recebe uma oferta substancial de água afluente de fora do seu limite. Tradicionalmente, a água subterrânea tem sido considerada como "protegida" pelo subsolo. No entanto, a disposição de poluentes estáveis de atividades humanas em áreas favoráveis de 
infiltração poderia causar esta contaminação dos recursos, colocando em risco a saúde da população e a sustentabilidade da reserva. Assim, é proposto se avaliar o risco de contaminação das águas subterrâneas da RBL e AI, através da sobreposição de um mapa de vulnerabilidade do aquífero usando o método GODS com um inventário de carga poluente utilizando o método POSH e, dessa forma, priorizar medidas de proteção deste recurso. Verificou-se que, na maior parte da área de estudo, não há perigo de contaminação das águas subterrâneas, no entanto, foram encontradas áreas de risco moderado e elevado para as quais foram propostas medidas para evitar a deterioração da qualidade dos recursos hídricos subterrâneos.

Palavras-chave: águas subterrâneas, aquíferos, contaminação das águas subterrâneas, sistemas de informação geográfica.

\title{
Evaluation of contamination risk of aquifers of the Limoncocha Biological Reserve, Ecuadorian Amazon Region
}

\begin{abstract}
Groundwater is one of the main supply sources of the inhabitants of Limoncocha and Pompeya parishes, located in the Ecuadorian Amazon region. The Limoncocha Biological Reserve (Spanish acronym, "RBL") is located in this region, and its area of influence (AI) has an underground ecosystem which receives a substantial supply of water from external flows. Traditionally, groundwater has been considered "protected" by the subsoil. However, the disposal of stable pollutants from human activities in areas favorable to infiltration could cause the contamination of this resource, risking the health of the population and the reserve's sustainability. Consequently, it was proposed that an assessment be done of the risk of groundwater contamination of the RBL and AI by overlapping the vulnerability map of the aquifer using the GODS method with an inventory of pollutant loads by the POSH method. In this way, measures for the protection of this resource might be prioritized. In most of the study area there was no danger of groundwater contamination. Nevertheless, areas of moderate and high risk were found. Measures to prevent the deterioration of the underground water quality were therefore proposed.
\end{abstract}

Keywords: aquifers, geographic information systems, groundwater, groundwater contamination.

\section{INTRODUCCION}

En un perfil de subsuelo normalmente existen dos zonas con características hidráulicas diferentes, la zona no saturada donde los poros del terreno están ocupados por agua y aire, y la zona saturada cuyos poros están totalmente llenos de agua originando acuíferos, que son formaciones geológicas capaces de almacenar y trasmitir agua subterránea en volúmenes apreciables (Collazo y Montaño, 2012).

El recurso hídrico subterráneo, al estar protegido por los perfiles del subsuelo, ha sido considerado como un suministro de agua económico y confiable para el consumo humano, tanto en el ámbito urbano como rural. Sin embargo, la generación de cargas contaminantes por actividades antrópicas, que excedan la capacidad natural de atenuación del suelo y estratos subyacentes, y cuya disposición se realice sobre el terreno, podría causar que este recurso se contamine sin posibilidad de recuperación alguna, debido a la inviabilidad técnica y económica de su tratamiento (Schmoll et al., 2006).

El concepto de vulnerabilidad de acuíferos ha sido discutido por diferentes hidrogeólogos:, para Vrba y Zaporozec (1994) se refiere a "una propiedad intrínseca del sistema de agua 
subterránea que depende de la sensibilidad del mismo a los impactos humanos y/o naturales"; Foster (1987) afirma que "la vulnerabilidad del acuífero a la contaminación, representa las características intrínsecas que determinan su susceptibilidad a ser adversamente afectado por una carga contaminante impuesta" y según Custodio (1996) "la vulnerabilidad a la polución expresa la incapacidad del sistema para absorber las alteraciones, tanto naturales como artificiales".

Por todo ello se puede diferenciar una vulnerabilidad intrínseca de una específica. La vulnerabilidad intrínseca hace referencia a la predisposición que tiene un acuífero a la contaminación, cuantificada únicamente en términos de sus propiedades hidrogeológicas, y a la capacidad de atenuación de los estratos por encima de la zona saturada, sin hacer referencia a contaminantes específicos; se trata de un parámetro relativo y adimensional. La vulnerabilidad específica en cambio se refiere al peligro de deterioro en relación con una carga contaminante específica.

En el mundo se han realizado numerosas investigaciones sobre la vulnerabilidad de los acuíferos, especialmente en América Latina y el Caribe (Duarte et al., 2016; Díaz et al., 2009; Cutrim et al., 2010; Löbler el al., 2015; Batista et al., 2017; Albarado et al., 2016). La literatura acerca de la determinación del peligro a la contaminación, también es abundante (Pinheiro et al., 2015; Jiménez et al., 2016; Durán et al., 2016; Solís et al., 2014; González et al., 2013; Lisboa et al., 2016).

En el Ecuador, importantes fuentes de agua subterránea se encuentran en acuíferos de la Amazonía (Buckalew et al., 1998), pero su calidad se ha visto afectada por la práctica de actividades antrópicas potencialmente contaminantes para este recurso. Por ejemplo, en Sucumbíos y Orellana donde gran parte del agua "potable" proviene de pozos profundos, el derrame de cerca de 16,8 millones de galones de petróleo durante la operación de Texaco, ha puesto en duda la calidad del agua subterránea en estas provincias, haciendo que su consumo se añada a las principales causas de enfermedad entre la población (Serrano, 2013).

En el país únicamente existen trabajos relativos a la vulnerabilidad de los acuíferos como el "Análisis Comparativo de la Vulnerabilidad del Acuífero Norte de Quito" realizado por Coello y Galárraga Sánchez (2002), en el que tras una modelación de las características físicas del acuífero de estudio se examinan distintas metodologías de zonificación de la vulnerabilidad, o el estudio realizado por Ribeiro et al. (2017) en el que se aplica un índice de susceptibilidad a la contaminación agrícola difusa en el acuífero de Daule en la provincia de Guayas. Para el sitio del proyecto sólo se dispone de estudios sobre las características geológicas y litológicas del terreno, propiedades hidrogeológicas como la permeabilidad (Ecuador, 2012) y análisis fisicoquímicos del agua subterránea realizados por Armas y Lasso (2011), pero ninguno enfocado a proteger la calidad de este recurso.

Todo ello conduce a la necesidad de proteger la calidad del agua subterránea con un enfoque hacia un adecuado uso del suelo, en el que se establezca dónde es posible realizar distintas actividades, como agricultura, usos industriales, ubicaciones de cementerios, lagunas de efluentes, entre otras.

Por consiguiente, en la presente investigación se propuso evaluar el peligro de contaminación del agua subterránea de la Reserva Biológica Limoncocha (RBL) y su Área de Influencia (AI), mediante la superposición de un mapa de vulnerabilidad del acuífero a la contaminación, con uno del inventario de cargas contaminantes al subsuelo con el objetivo de mejorar la planificación y el ordenamiento territorial, en este caso, con énfasis en la protección de acuíferos. En relación a todo lo anterior se plantea la hipótesis de que la superposición del mapa de vulnerabilidad a la contaminación de los acuíferos con el mapa de inventario de carga contaminante, permite estimar las zonas de peligro a la contaminación de las aguas subterráneas. 


\section{MATERIALES Y MÉTODOS}

\section{1. Área de estudio}

La zona de estudio forma parte de la cuenca amazónica ecuatoriana, en la subcuenca del Río Capucuy, tributario del río Napo y está comprendida por la RBL y su AI. Tiene una extensión de 11.034 ha, la misma que ocupa en su mayor parte la provincia de Sucumbíos, parroquia Limoncocha, mientras que la superficie restante pertenece en la provincia de Orellana, parroquia Pompeya. Según el Instituto Nacional de Estadísticas y Censos (2010), en Limoncocha el $43.02 \%$ de los habitantes consume agua proveniente de pozos subterráneos, mientras que en Pompeya lo hace el 5,61\% de ellos.

La investigación se desarrolló con base en una metodología para establecer zonas prioritarias de protección del agua subterránea en función al mapeo de su peligro a la contaminación. La cartografía base para el estudio se obtuvo del Instituto Geográfico Militar (2012), Ministerio de Agricultura, Ganadería, Acuacultura y Pesca (Ecuador, 2012) y Ministerio del Ambiente (Ecuador, 2013). Esta metodología está compuesta, por el método GODS y el método POSH (Foster et al., 2002).

\subsection{Vulnerabilidad del acuífero mediante el método GODS}

Foster et al. (2002) establecen que la vulnerabilidad de un acuífero a la contaminación depende de la combinación de cuatro parámetros cuyas iniciales le confieren el nombre de método GODS (Tabla 1). El método GODS evalúa básicamente dos componentes de la vulnerabilidad: la inaccesibilidad hidráulica del contaminante y la capacidad de autodepuración que tiene la zona no saturada. El primer componente está relacionado con el tipo de acuífero, atendiendo a la presión a la que se encuentra el agua subterránea (el grado de confinamiento hidráulico como parámetro G) y con la profundidad a la que se encuentra el agua subterránea o el techo del acuífero en el caso de los confinados (parámetro D). El parámetro O responde al componente de la capacidad de atenuación del contaminante debido al sustrato, en función del tamaño de granos o fisuras y a las propias características litológicas de los estratos. Así mismo, el parámetro $\mathrm{S}$ tiene en cuenta la forma de atenuación del contaminante y su eliminación debido al contenido de materia orgánica.

Dicho método permite clasificar a la vulnerabilidad en nula, despreciable, baja, media, alta y extrema.

Tabla 1. Parámetros del método GODS.

\begin{tabular}{|c|c|c|}
\hline Sigla & Significado & Basado en \\
\hline G & Grado de confinamiento hidráulico & Tipo de acuífero según estructura. \\
\hline $\mathrm{O}$ & Ocurrencia del sustrato suprayacente & $\begin{array}{l}\text { Características litológicas: } \\
\text { consolidación, fracturamiento, } \\
\text { porosidad y permeabilidad }\end{array}$ \\
\hline $\mathrm{D}$ & Distancia al nivel de agua subterránea & $\begin{array}{l}\text { Profundidad del nivel de agua en } \\
\text { acuíferos libres o profundidad al } \\
\text { techo de acuíferos confinados }\end{array}$ \\
\hline $\mathrm{S}$ & Tipo de suelo & $\begin{array}{l}\text { Textura y contenido en materia } \\
\text { orgánica }\end{array}$ \\
\hline
\end{tabular}

Fuente: Adaptado de Foster et al. (2002).

El mapa de vulnerabilidad de la RBL y su AI se generó aplicando el sistema de indexación GOD, planteado por Foster e Hirata (1988), con una modificación que incorpora el parámetro 
S para considerar la capacidad de atenuación del suelo. Este sistema permitió estimar el índice final integrado de vulnerabilidad de acuíferos GODS (IVGODS), aplicando la Ecuación 1.

$$
\text { IVGODS }=G \cdot O \cdot D \cdot S
$$

Con base en esta ecuación se llevó a cabo una serie de etapas establecidas por Foster e Hirata (1988), previas a la determinación de la vulnerabilidad del acuífero objeto de estudio, que fueron las siguientes:

- Identificar el grado de confinamiento hidráulico del acuífero y asignarle un índice en una escala de 0,0 a 1,0

- Especificar las características del sustrato suprayacente a la zona saturada del acuífero y asignar un índice de 0,4 a 1,0

- Estimar la distancia al nivel del agua en acuíferos libres o la profundidad al techo en acuíferos confinados y asignar un índice comprendido entre 0,6 y 1,0.

- Determinar el tipo de suelo y asignar un índice en una escala de 0,5 a 1,0.

La asignación de los índices a cada parámetro se hizo en función al esquema modificado por Foster et al. (2002) (Figura 1).

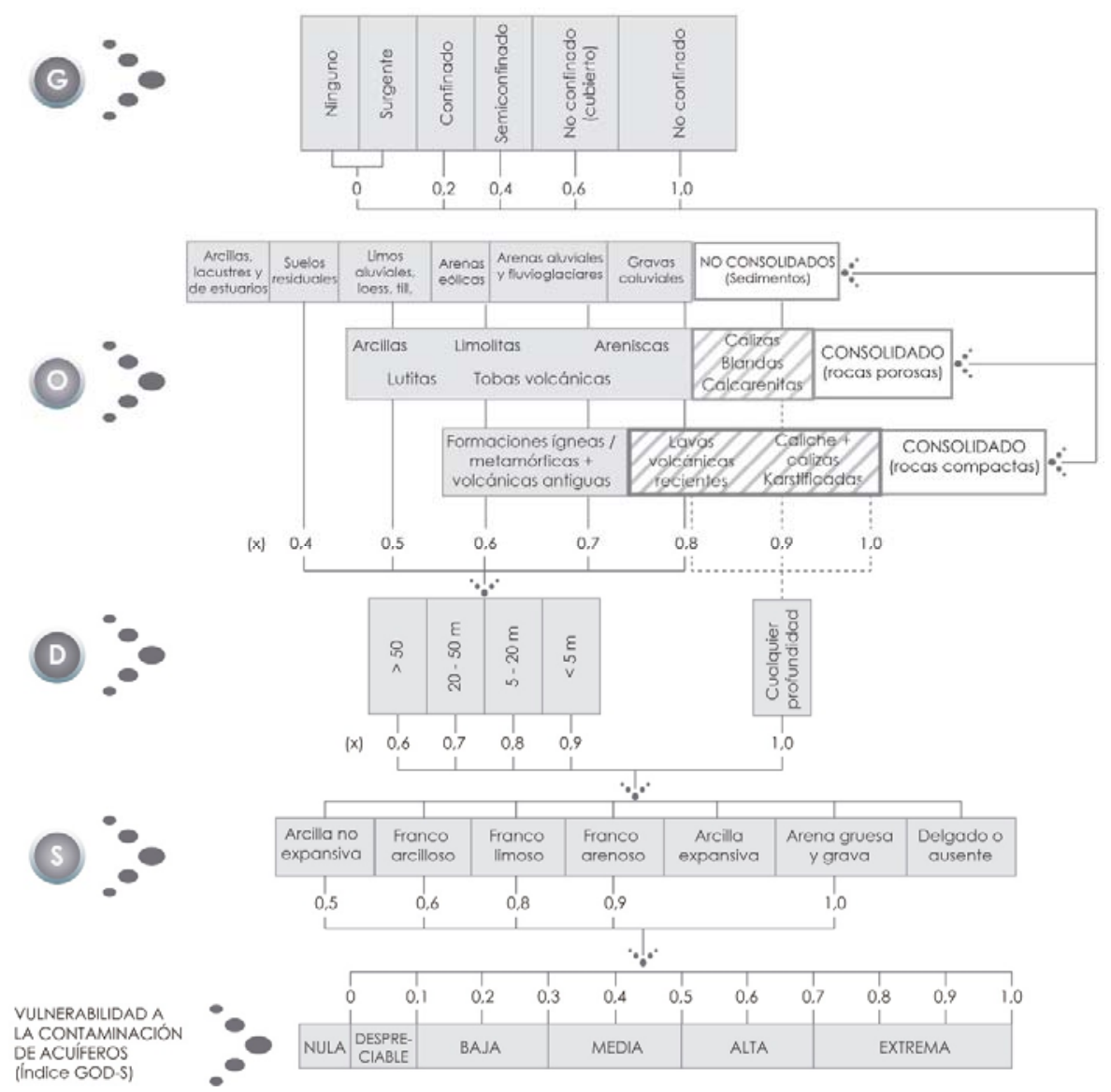

Figura 1. Método GODS para la evaluación de la vulnerabilidad a la contaminación de acuíferos.

Fuente: Adaptado de Foster et al. (2002).

Finalmente, se utilizó el software QGIS 2.14.3 (Quantum GIS, 2016) a fin de convertir la ecuación 1 en una expresión de algebra de mapas, de esta manera poder operar los parámetros G, O, D y S, generando así el mapa de vulnerabilidad de la RBL y su AI. 


\subsection{Mapeo del inventario de cargas contaminantes mediante el método POSH}

Existen contaminantes que por su composición química son móviles y persistentes en el subsuelo, lo que hace que no puedan ser degradados eficazmente durante su trasporte hacia el acuífero, y que puedan llegar a contaminar el agua subterránea. En este sentido, todas las fuentes de contaminación inventariadas en la RBL y su AI, están asociadas a diferentes agentes contaminantes para este recurso, tal como se indica a continuación (Foster et al., 2002):

- Fuentes difusas: los sistemas de saneamiento generan nitratos y microorganismos patógenos; mientras que las prácticas agrícolas están asociadas a fertilizantes (nitratos), pesticidas (hidrocarburos halogenados, fenoles, arsénico) y organismos fecales.

- Fuentes lineales: las rutas de trasporte están asociadas a accidentes que involucran el derrame de sustancias peligrosas; las tuberías de petróleo pueden ser fuente de hidrocarburos aromáticos; y los cursos de agua superficial contaminada están asociadas principalmente a cargas orgánicas y a elevada salinidad.

- Fuentes puntuales: se incluyen industrias que según su actividad pueden generar hidrocarburos, solventes orgánicos, metales pesados. La disposición de residuos sólidos genera amonio, salinidad, hidrocarburos halogenados, metales pesados. Las lagunas de efluentes pueden contener microorganismos patógenos, metales pesados, nutrientes y solventes orgánicos. Los cementerios están asociados a cargas contaminantes microbiológicas; y la explotación petrolera puede generar sales e hidrocarburos aromáticos.

Para la identificación, georreferenciaron y caracterizaron de cada fuente de contaminación se llevaron a cabo campañas de campo, empleándose diferentes técnicas (muestreo, búsqueda y entrevista) dependiendo de la fuente a inventariar.

La categorización del potencial de carga contaminante se realizó aplicando el método POSH, que por sus siglas en inglés, propone categorizar cada carga sobre la base de dos características que, de acuerdo a Foster et al. (2002), son:

- El origen del contaminante (Pollutant Origin) asumiendo que este sea persistente y móvil en el subsuelo y que su presencia se asocie al tipo de actividad antrópica de la que proviene.

- El incremento de la recarga de agua natural al acuífero (Surcharge Hydraulically) en base al uso del agua en la actividad relacionada.

La evaluación de cada fuente en función a las características propuestas por el método POSH, permitió generar tres niveles cualitativos de potencial de generación de una carga contaminante al subsuelo: reducido, moderado y elevado.

Para el mapeo del inventario se generaron y exportaron bases de datos de fuentes de contaminación difusa, lineal y puntual a la plataforma de QGIS y se asignó una simbología específica a cada tipo de fuente y a su potencial, creándose de esta manera un mapa integrado del inventario de cargas contaminantes al subsuelo de la RBL y su AI. 


\subsection{Evaluación del peligro de contaminación del agua subterránea}

Una vez obtenido el mapa de vulnerabilidad y el de inventario, se generó una matriz que permitió asignar a cuatro conjuntos de números diferentes, la calificación de peligro alto, moderado, bajo o ausente, representado en la Figura 2.

\begin{tabular}{|c|c|c|c|c|}
\hline Vulnerabilidad & Nula & Baja & Media & Alta \\
\hline Ninguno & Ausente & Ausente & Ausente & Ausente \\
\hline Reducido & Ausente & Bajo & Bajo & Moderado \\
\hline Moderado & Ausente & Moderado & Moderado & Alto \\
\hline Elevado & Ausente & Moderado & Alto & Alto \\
\hline
\end{tabular}

\begin{tabular}{|c|c|c|c|c|}
\hline Potencial & 5 & 7 & 8 & 9 \\
\hline 1 & 5 & 7 & 8 & 10 \\
\hline 2 & 10 & 14 & 16 & 18 \\
\hline 3 & 15 & 21 & 24 & 27 \\
\hline 4 & 20 & 28 & 32 & 36 \\
\hline
\end{tabular}

Figura 2. Matriz para la calificación del peligro a la contaminación del agua subterránea.

Con la ayuda de la plataforma QGIS, se crearon campos para asignar los valores indicados en la Figura 3, tanto en los mapas de vulnerabilidad como en el de inventario de cargas contaminantes. Finalmente utilizando las herramientas de álgebra de mapas, se generó el mapa de peligro de contaminación de la RBL y su AI.

\section{RESULTADOS Y DISCUSION}

En todos los mapas realizados siguiendo la metodología GODS, se descartó el territorio ocupado por la laguna de Limoncocha, laguna Negra y los ríos Napo, Chicta y Jivino, por tratarse de cuerpos de agua superficial cuya área ocupa el 17\% de la zona de estudio.

Sobre el parámetro $\mathrm{G}$ se consideró a todo el acuífero como no confinado, abarcando el $83 \%$ de la zona investigada. En el parámetro O, la litología predominante correspondió a arenas aluviales con el $43 \%$, seguida de gravas coluviales, areniscas y lutitas con el $40 \%$. En el parámetro D se determinó que el mayor porcentaje de agua subterránea se encuentra entre 5 y $20 \mathrm{~m}$ de profundidad, correspondiente al $45 \%$ del área de estudio, seguido del $25 \%$ con una distancia menor a $5 \mathrm{~m}$, mientras que en el $6 \%$ del terreno se encuentra entre $>20$ y $26,21 \mathrm{~m}$ de profundidad y en el $7 \%$ se desconoce. En el parámetro S, la superficie cartográfica indica que más de la mitad del área de estudio tiene un suelo de textura franco-limosa con el 52\%, arcillosa con el 22\% y franco arenosa con el 10\%. En la Figura 3 se esquematizan los mapas elaborados para cada parámetro, con los índices asignados según sus características intrínsecas a la contaminación.

A partir del producto de estos cuatro parámetros, se pudo generar el mapa de vulnerabilidad a la contaminación del acuífero de la RBL y su AI, tal como se aprecia en la Figura 4. El índice de vulnerabilidad está comprendido entre 0 y 0.7 , resultando tres categorías diferentes: en la mayoría de la zona de estudio, el 44\%, corresponde a una vulnerabilidad media, que incluye las comunidades de Playayacu, Río Jivino y Jacinto Rodríguez, la vulnerabilidad alta ocupa el 17\% sobre la que se asientan las comunidades de Limoncocha, Capucuy, Puerto palo y Pompeya, mientras que en el $15 \%$ del territorio fue baja, un $17 \%$ nula y el $7 \%$ de vulnerabilidad desconocida al no disponer datos para las zonas perimetrales del área de influencia. 


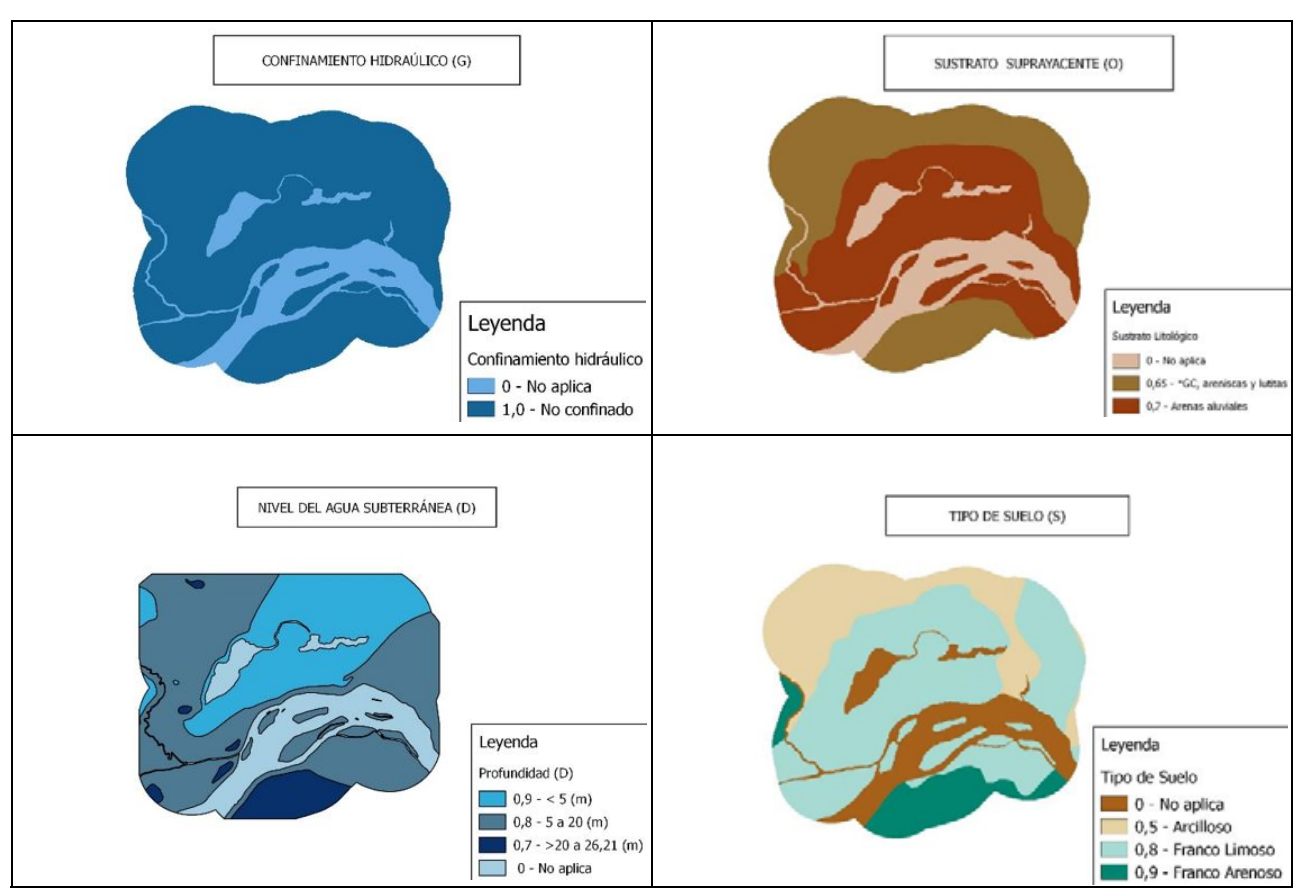

Figura 3. Mapas paramétricos indexados de acuerdo al método GODS.

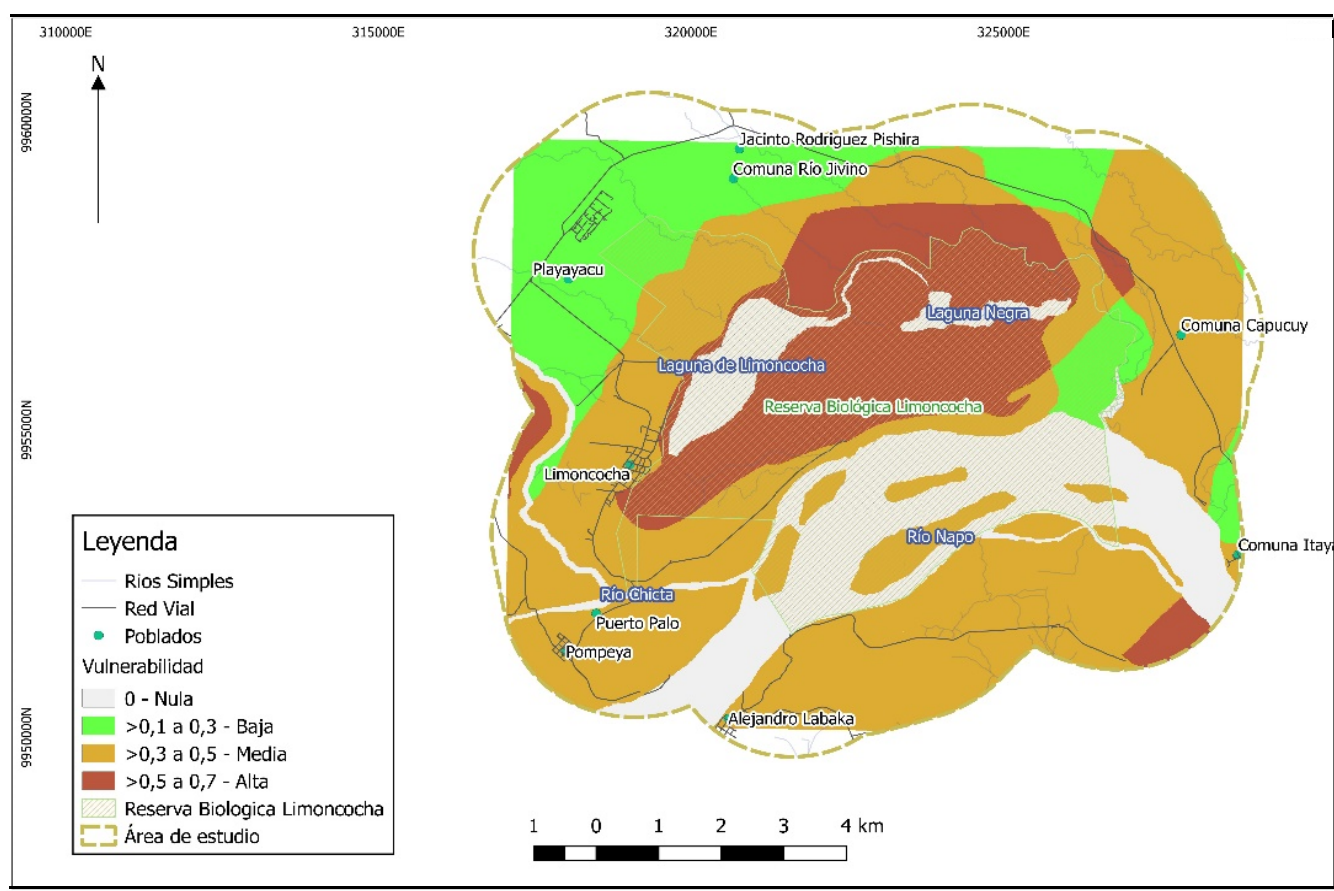

Figura 4. Mapa de la vulnerabilidad a la contaminación del acuífero del área de estudio.

En la Tabla 2 se presenta el total de cada tipo de fuente inventariada y el número de cargas contaminantes categorizadas según su nivel de potencial. Adicionalmente se estableció el potencial global de generación de cargas contaminantes al subsuelo de la zona de estudio, según el cual de 282 fuentes de contaminación identificadas, 218 tuvieron un potencial moderado, 36 elevado y 28 reducido. 
Tabla 2. Categorización de fuentes contaminantes.

\begin{tabular}{lcccc}
\hline \multirow{2}{*}{ Fuente de contaminación } & \multicolumn{3}{c}{ Potencial } & \multirow{2}{*}{ Total } \\
\cline { 2 - 4 } Saneamiento & Reducido & Moderado & Elevado & $\mathbf{1 5 5}$ \\
Prácticas agrícolas & 0 & 155 & 0 & $\mathbf{3 5}$ \\
Rutas de transporte & 2 & 10 & 23 & $\mathbf{3}$ \\
Oleoductos & 0 & 3 & 0 & $\mathbf{1}$ \\
Aguas superficiales contaminadas & 0 & 0 & 1 & $\mathbf{8}$ \\
Industrias & 1 & 5 & 2 & $\mathbf{1}$ \\
Resíduos sólidos & 1 & 0 & 0 & $\mathbf{4 4}$ \\
Lagunas efluentes & 0 & 44 & 0 & $\mathbf{2 4}$ \\
Cementerios & 23 & 1 & 0 & $\mathbf{1}$ \\
Explotación petrolera & 1 & 0 & 0 & $\mathbf{1 0}$ \\
\hline Total Global & 0 & 0 & 10 & $\mathbf{2 8 2}$ \\
\hline
\end{tabular}

Las fuentes de contaminación difusa, lineal y puntual identificadas, fueron georreferenciadas dentro de la zona de estudio a través de una simbología específica para cada tipo de fuente y de potencial de carga contaminante, dando lugar al mapa del inventario del potencial de cargas contaminantes al subsuelo de la RBL y su AI, constituido en la Figura 5.

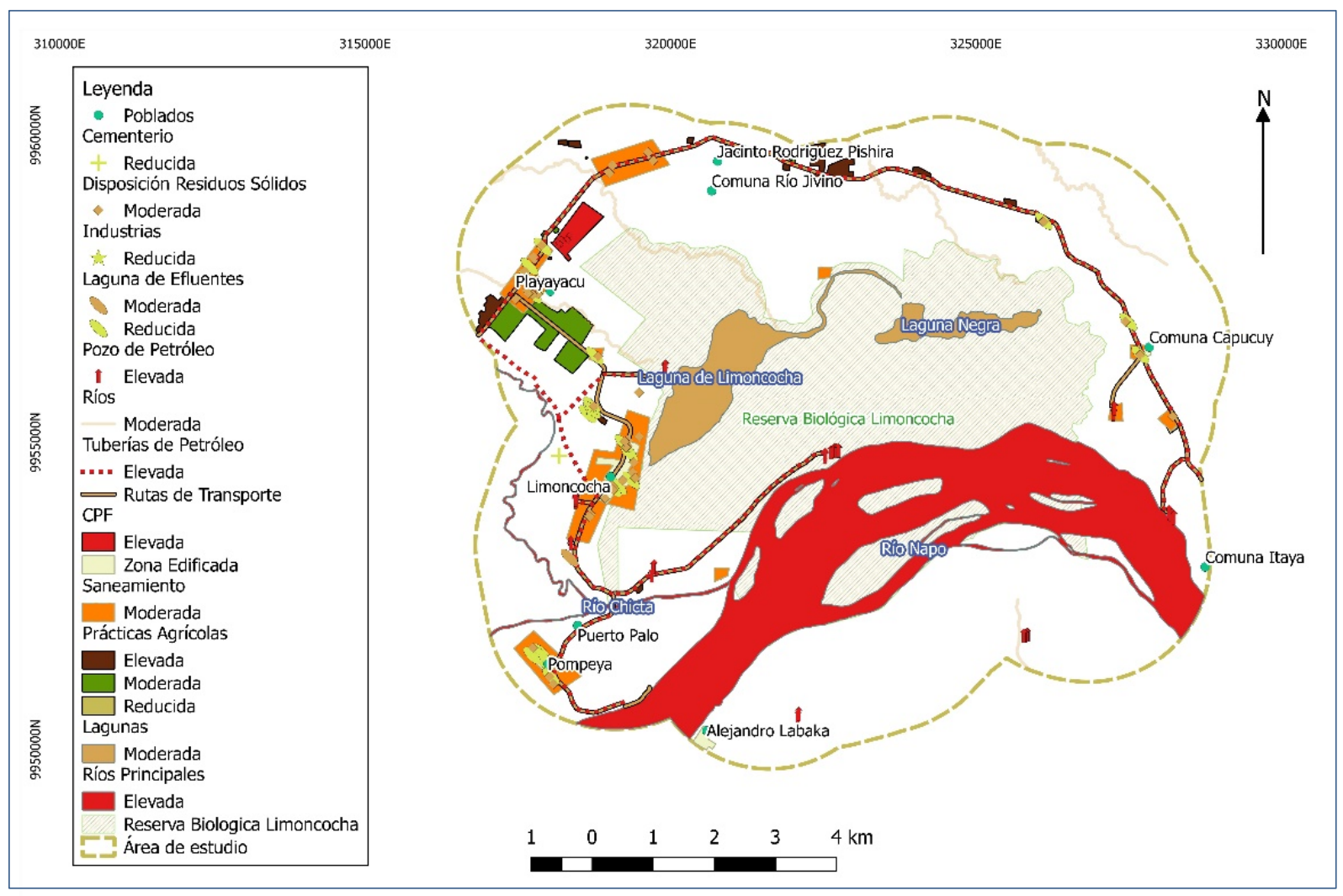

Figura 5. Mapa del inventario del potencial de cargas contaminantes al subsuelo del área de estudio.

Producto de la superposición del mapa de vulnerabilidad con el mapa del inventario de cargas contaminantes, se obtuvo una representación cartográfica del peligro de contaminación del agua subterránea de la RBL y su AI (Figura 6). 


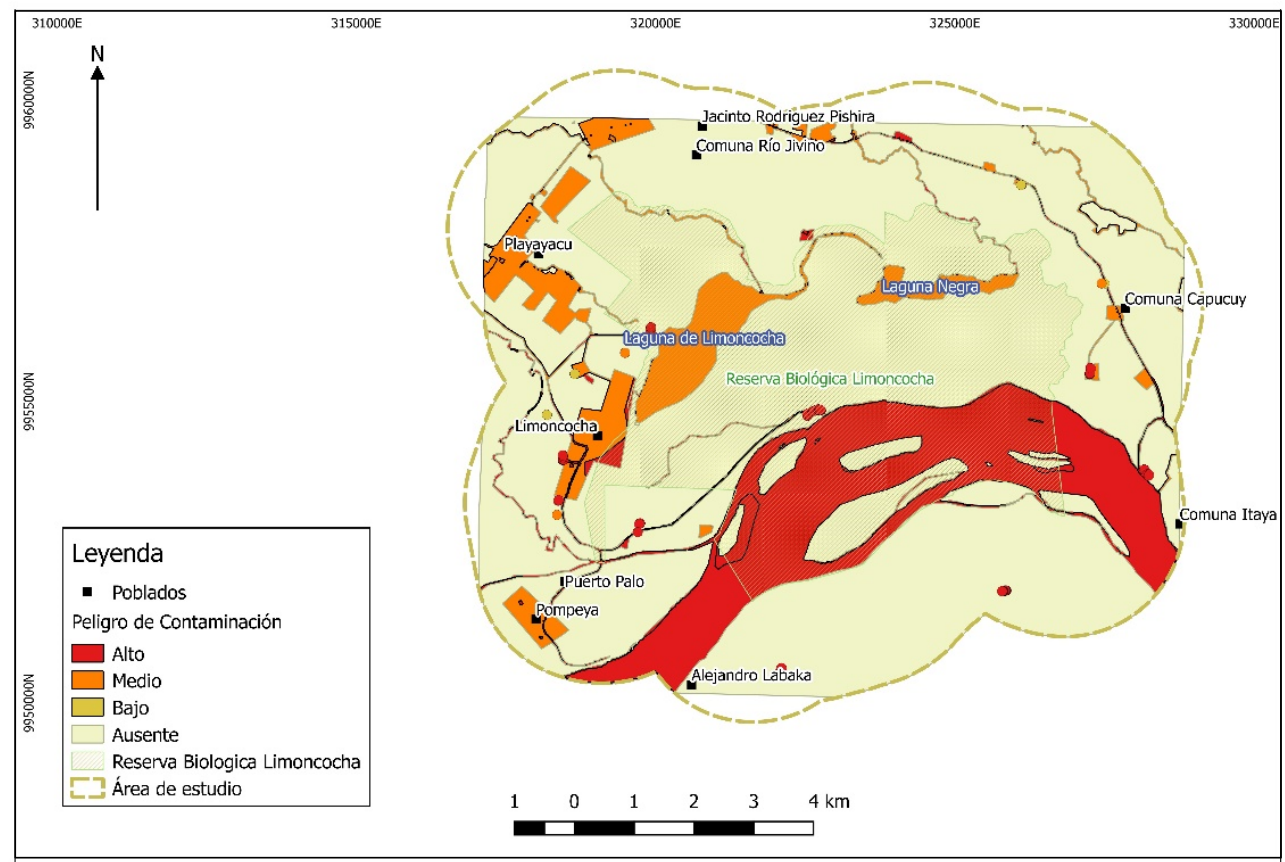

Figura 6. Mapa del peligro de contaminación del agua subterránea del área de estudio.

Se determinó que el 72\% del área de evaluación, que representa 7.905 ha, dicho peligro se encuentra ausente, en el 14\% del territorio unas 1.539,3 ha correspondientes a los ríos con alta carga contaminante como el Napo, los pozos y tuberías de petróleo, y las zonas agrícolas con prácticas no sostenibles el peligro es alto, mientras que en el 7\% que ocupa 808 ha en las zonas de cuerpos hídricos como la Laguna de Limoncocha, la laguna Negra o en las superficies donde se asientan las distintas comunidades es medio y en el 7\% es desconocido.

No obstante aunque existen zonas de bajo peligro de contaminación, su valor fue despreciable y en las zonas ocupadas por cuerpos de agua superficial, pese a tener una vulnerabilidad nula, se les asignó un nivel de peligro de contaminación análogo a su potencial de carga contaminante; esto es debido a que no se puede catalogar una vulnerabilidad a dichos cuerpos de agua (al no ser formaciones geológicas) pero es posible determinar la calidad química de sus aguas, por lo que la carga contaminante impuesta será la que predomine en el peligro a la contaminación de los acuíferos.

\section{CONCLUSIONES}

Las características naturales intrínsecas del acuífero de la RBL y su AI le confieren, en su mayoría, una vulnerabilidad a la contaminación media. No obstante, también existe una importante cobertura de vulnerabilidad alta, ubicada principalmente dentro de los límites de la RBL donde la distancia al nivel del agua subterránea es menor.

En el inventario de cargas contaminantes al subsuelo de la RBL y su AI, gran parte de las fuentes identificadas generan una carga de potencial moderado, existiendo también cargas de potencial elevado provenientes de las instalaciones petroleras, de algunas prácticas agrícolas y del agua superficial contaminada de los ríos Napo y Jivino.

Se concluye que en la mayoría del área de estudio (72\%), no existe peligro de contaminación del agua subterránea, debido únicamente a la ausencia de cargas contaminantes impuestas, ya que no se encontraron zonas de vulnerabilidad despreciable. Por otra parte, se hallaron zonas de alto peligro asociadas a la disposición de cargas contaminantes de potencial elevado en zonas de vulnerabilidad alta y media.

\section{IPABH}

Rev. Ambient. Água vol. 12 n. 4 Taubaté - Jul. / Aug. 2017 
En el Plan de Manejo de la RBL elaborado por Armas y Lasso (2011), consta que el consumo de agua subterránea es una de las principales causas de enfermedad entre la población, ya que la calidad del agua subterránea proveniente del acuífero investigado, podría verse afectada por la práctica de actividades antrópicas potencialmente contaminantes en esta zona, como son: la agropecuaria, la explotación petrolera, el turismo y los servicios, siendo la más ejercida, tanto en Limoncocha como en Pompeya, la agricultura con el $40 \%$ y el $43 \%$, respectivamente.

Es posible que la predominancia de las áreas con ausencia de peligro en la zona de estudio coincida con el hecho de que gran parte de la misma pertenezca al Sistema Nacional de Áreas Protegidas (SNAP) y que un importante porcentaje de los habitantes de Limoncocha y Pompeya consuman agua de pozos profundos. Sin embargo, dentro de los límites de la RBL, pese a ser un área protegida, se llevan a cabo actividades productivas de tipo turístico en las cabañas "Supay-Cocha" y de explotación petrolera en el pozo "Laguna A", las cuales merecen una gestión prioritaria respecto a la generación de cargas contaminantes al subsuelo.

Se recomienda tener en cuenta la zonificación del peligro de contaminación del agua subterránea de la RBL y su AI dentro de los planes de desarrollo y ordenamiento territorial de las parroquias de Limoncocha y Pompeya, a fin de asignar un uso adecuado del suelo, que evite primordialmente la ubicación de cargas contaminantes de potencial moderado y elevado en zonas de vulnerabilidad alta.

Respecto al saneamiento in situ, es importante eliminar la carga contaminante generada en ambas parroquias, y las fugas de la red de alcantarillado en Limoncocha ya que atentan contra la calidad del agua subterránea en la zona. Para ello se propone como solución a corto plazo, impermeabilizar el sitio de descarga in situ y evacuar periódicamente los pozos negros, letrinas y tanques sépticos, mediante un sistema de aspiración. A largo plazo se debe optar por la construcción de la red de cloacas en Pompeya y por el rediseño y ampliación de la red de alcantarillado en Limoncocha, cuya descarga debe ser en una zona de baja vulnerabilidad.

En cuanto a las prácticas agrícolas se recomienda que se reemplace el uso de agroquímicos por otros de contenido orgánico y que adicionalmente, se cultive en zonas de baja vulnerabilidad (Figura 2).

En los ríos Napo y Jivino se debe evitar el consumo debido a la baja calidad de sus aguas y por otra parte el vertido de efluentes líquidos a dichos cauces que empeoren la carga contaminante impuesta. Se propone que se invierta en su recuperación, se controle la descarga de efluentes hacia ellos y se monitoree periódicamente su calidad.

La recomendación de mayor importancia es orientada hacia la actividad de los campos petroleros. En todas sus instalaciones se debe realizar un control y mantenimiento periódico que evite el derrame de hidrocarburos en el terreno. Además, aprovechando las técnicas de perforación direccionada, se deben ubicar a las plataformas petroleras en zonas de baja vulnerabilidad del acuífero, reduciendo así al mínimo el peligro de contaminación del agua subterránea de la RBL y su AI.

Finalmente, deben ser abordados estudios sobre perímetros de protección para captaciones subterráneas, a fin de evitar la construcción de pozos cerca de fuentes de contaminación potencial. Asimismo, se deben eliminar las cargas contaminantes provenientes del saneamiento in situ y agricultura en las cabañas "Supay-Cocha" y de la explotación petrolera en el pozo "Laguna A".

\section{AGRADECIMIENTOS}

Financiamiento: Dirección de Investigación e Innovación de la Universidad Internacional SEK. Ministerios del Ambiente (Limoncocha). A nuestros compañeros Juan Carlos Navarro y 
Fabio Villalba por sus comentarios y sugerencias. Al personal de la Estación Biológica Limoncocha por el apoyo logístico.

\section{REFERENCIAS}

ARMAS, M. Y.; LASSO, S. Plan de manejo de la reserva biológica Limoncocha. Quito: Ministerio del Ambiente, 2011. Disponible en: https://goo.gl/ymd9uN . Acceso: Sep. 2016.

BATISTA, C. S. P.; GESUALDO, G. C.; LEITE, P. C. C.; LASTORIA, G.; GABAS, S. G.; CAVAZZANA, G. H. et al. Aplicação do método GOD para avaliação de vulnerabilidade de aquífero livre em bacia hidrográfica. Águas Subterrâneas, supl., 2016.

BUCKALEW, J.; REED, P.; SCOTT, L.; JAMES, M. Evaluación de los recursos de agua del Ecuador. Ecuador: Centro de Ingeniería y Topografía, Cuerpos de Ingenieros de los Estados Unidos de América, 1998. 90p.

COEllO RUBIO, X.; GALÁRRAGA SÁNCHEZ, R. Análisis comparativo de la vulnerabilidad del acuífero norte de Quito. Águas Subterrâneas, supl., 2002

COLlaZO, C. M. P.; MONTAÑO, X. J. Manual de agua subterránea. Montevideo: Ministerio de ganadería agricultura y pesca dirección general de desarrollo rural, 2012. p. 978-9974.

CUSTODIO, E. Consideraciones sobre el concepto de vulnerabilidad de los acuíferos a la polución. En: SEMINARIO HISPANO-ARGENTINO SOBRE TEMAS ACTUALES DE HIDROLOGÍA SUBTERRÁNEA, 2., 20-23 septiembre 1995, San Miguel de Tucumán. Resumos... Tucumán: Facultad de Ciencias Naturales; Instituto Miguel Lillo; Universidad Nacional de Tucumán, 1996. p. 99-122. (Serie correlación geológica, v. 11)

CUTRIM, A. O.; CAMPOS, J. E. G. Avaliação da vulnerabilidade e perigo à contaminação do Aqüífero Furnas na Cidade De Rondonópolis (MT) com aplicação dos métodos GOD e POSH. Geociências, v. 29, n. 3, p. 401-411, 2010.

DÍAZ, E. L.; ROMERO, E. C.; BOSCHETTI, N. G.; DUARTE, O. C. Vulnerabilidad del agua subterránea en la cuenca del Arroyo Feliciano. Boletín Geológico y Minero, v. 120, n. 4, p. 533-542, 2009.

DUARTE, M. L.; ZANCHI, F. B.; NEVES, J. R. D.; COSTA, H. S.; JORDÃO, W. H. C. Vulnerabilidade à contaminação das águas subterrâneas no município de Humaitá, Amazonas, Brasil. Revista Ambiente \& Água, v. 11, n. 2, p. 402-413, 2016. http://dx.doi.org/10.4136/ambi-agua.1797

DURÁN, A.; PARIS, M.; MAITRE, M. I.; MARINO, F. Diagnóstico ambiental en la zona del cinturón hortícola de la ciudad de Santa Fe. Revista de la Facultad de Ciencias Agrarias, v. 48, n. 1, p. 129-143, 2016.

ECUADOR. Ministerio de Agricultura, Ganadería, Acuacultura y Pesca - MAGAP. Mapas temáticos del Ecuador. Geoportal MAGAP, 2012.

ECUADOR. Ministerio del Ambiente. Mapa de cobertura y uso de la tierra. Escala 1:100.000. Geoportal, MAE, 2013. 
FOSTER, S. Fundamental concepts in aquifer vulnerability, pollution, risk and protection strategy. In: VAN DUIJVENBOODEN, W.; VAN WAEGENINGH, H. G. (Eds.). Vulnerability of soil and groundwater to pollutants. The Hague: TNO Committee on Hydrological Research, 1987. v. 38. p. 69-86.

FOSTER, S.; HIRATA R.; GOMES, D’ELIA D. M.; PARIS, M. Protección de la calidad del agua subterránea: guía para empresas de agua, autoridades municipales y agencias ambientales. Washington D.C.: Banco Mundial, 2002.

FOSTER, S.; HIRATA, R. Determinación del riesgo de contaminación de aguas subterráneas: una metodología basada en datos existentes. En Determinacion del riesgo de contaminacion de aguas subterraneas: una metodologia basada en datos existentes. [S.1.]: OPS, 1988.

GONZÁLEZ ESTÉVEZ, M.; SÁNCHEZ MUNGUÍA, V. Riesgo de contaminación del acuífero arroyo Alamar en Tijuana, Baja California. Región y sociedad, v. 25, n. 56, p. 103-126', 2013.

INSTITUTO GEOGRÁFICO MILITAR. Base Cartográfica Nacional, escala 1:50.000. Ecuador: IGN, 2012.

INSTITUTO NACIONAL DE ESTADÍSTICAS Y CENSOS - INEC. Base de datos del séptimo censo de la población y la vivienda. Quito, 2010.

JIMÉNEZ PEÑA, Y.; VIDAL OLIVERA, V. M.; GONZÁLEZ-ABREU FERNÁNDEZ, R.; VALDÉS GONZÁlEZ, L. A.; CASTRO CARRILlO, M. Potencial de carga contaminante en sector hidrogeológico de la provincia Ciego de Ávila. Ingeniería Hidráulica y Ambiental, v. 37, n. 2, p. 79-93, 2016.

LISBOA, E. G.; MENDES, R. L. R.; BELLO, L. A. L. Mapeamento do risco de contaminação das águas subterrâneas em zonas urbanas: proposta de um índice de perigo. Revista da Universidade Vale do Rio Verde, v. 14, n. 2, p. 238-252, 2016.

LÖBLER, C. A.; SILVA, J. L. S. DA. Vulnerabilidade à contaminação das águas subterrâneas do município de Nova Palma, Rio Grande do Sul, Brasil/Contamination vulnerability of groundwater in the municipality of Nova Palma, Rio Grande do Sul, Brazil. Revista Ambiente \& Água, v. 10, n. 1, p. 141-152, 2015. http://dx.doi.org/10.4136/ambiagua. 1390

PINHEIRO, R. J.; RAUBER, A. C. C.; NUMMER, A. V.; DA SILVA, J. L. S. Aplicação dos Métodos GOD e POSH para determinação da vulnerabilidade e perigo à contaminação dos aquíferos na cidade de Santa Maria-RS. Revista de Gestão de Água da América Latina, v. 12, n. 2, p. 61-79, 2015.

QUANTUM GIS DEVELOPMENT TEAM. Quantum GIS- Geographic Information System. Open Source Geospatial Foundation Project. 2016. Disponible: http://qgis.osgeo.org. Acceso: Jan. 2017

RIBEIRO, L.; PINDO, J. C.; DOMINGUEZ-GRANDA, L. Assessment of groundwater vulnerability in the Daule aquifer, Ecuador, using the susceptibility index method. Science of The Total Environment, v. 574, p. 1674-1683, 2017. https://doi.org/10.1016/j.scitotenv.2016.09.004 
SCHMOLL, O.; HOWARD, G.; CHILTON, J.; CHORUS, I. (Eds.). Protecting Groundwater for Health: managing the quality of drinking-water sources. Seattle: WHO; IWA, 2006. $678 \mathrm{p}$.

SERRANO, H. Caso Chevron-Texaco Cuando los pueblos toman la palabra. Quito: Corporación editora nacional, 2013. 118 p.

SOLÍS, H. M.; SÁNCHEZ, A. F.; SOLÍS, C. N.; CRUZ, A. G. Amenaza de contaminación del agua subterránea en el sector norte del acuífero Barva, Heredia, Costa Rica. Tecnología y Ciencias del Agua, v. 5, n. 6, p. 103-118, 2014.

VRBA, J.; ZAPOROZEC, A. Guidebook on mapping groundwater vulnerability. Heise: International Association of Hydrogeologist; International Contribitions to Hydrogeology, 1994. 131p. 